KYUNGPOOK Math. J. 55(2015), 429-438

http://dx.doi.org/10.5666/KMJ.2015.55.2.429

pISSN 1225-6951 eISSN 0454-8124

(c) Kyungpook Mathematical Journal

\title{
Coefficient Inequality for Transforms of Starlike and Convex Functions with Respect to Symmetric Points
}

\author{
Deekonda Vamshee Krishna* and Bollineni Venkateswarlu \\ Department of Mathematics, GIT, GITAM University, Visakhapatnam 530-045, \\ A.P., India \\ e-mail : vamsheekrishna1972@gmail.com and bvlmaths@gmail.com \\ ThoutredDy RAMrEDDY \\ Department of Mathematics, Kakatiya University, Warangal 506-009, T.S., India \\ e-mail : reddytr2@gmail.com
}

Abstract. The objective of this paper is to obtain sharp upper bound for the second Hankel functional associated with the $k^{t h}$ root transform $\left[f\left(z^{k}\right)\right]^{\frac{1}{k}}$ of normalized analytic function $f(z)$ when it belongs to the class of starlike and convex functions with respect to symmetric points, defined on the open unit disc in the complex plane, using Toeplitz determinants.

\section{Introduction}

Let $A$ denote the class of all functions $f(z)$ of the form

$$
f(z)=z+\sum_{n=2}^{\infty} a_{n} z^{n}
$$

in the open unit disc $E=\{z:|z|<1\}$. Let $S$ be the subclass of $A$ consisting of univalent functions. For a univalent function in the class $A$, it is well known that the $n^{\text {th }}$ coefficient is bounded by $n$. The geometric properties of these functions were determined by the study of their coefficient bounds. For example, the bound for the second coefficient of normalized univalent function readily yields the growth and distortion properties for univalent functions. The Hankel determinant of $f$ for

* Corresponding Author.

Received January 2, 2014; revised June 4, 2014; accepted October 30, 2014.

2010 Mathematics Subject Classification: 30C45, 30C50.

Key words and phrases: starlike and convex functions with respect to symmetric points, upper bound, second Hankel functional, positive real function, Toeplitz determinants. 
$q \geq 1$ and $n \geq 1$ was defined by Pommerenke [13] as

$$
H_{q}(n)=\left|\begin{array}{cccc}
a_{n} & a_{n+1} & \cdots & a_{n+q-1} \\
a_{n+1} & a_{n+2} & \cdots & a_{n+q} \\
\vdots & \vdots & \vdots & \vdots \\
a_{n+q-1} & a_{n+q} & \cdots & a_{n+2 q-2}
\end{array}\right|,\left(a_{1}=1\right) .
$$

This determinant has been considered by many authors in the literature. For example, Noor [11] determined the rate of growth of $H_{q}(n)$ as $n \rightarrow \infty$ for the functions in $S$ with bounded boundary. Ehrenborg [5] studied the Hankel determinant of exponential polynomials. In the recent years, several authors have investigated bounds for the Hankel determinant of functions belonging to various subclasses of univalent and multivalent analytic functions $[1,7,9,10,15]$. In particular for $q=2, n=1$ and $q=2, n=2$ when $\left(a_{1}=1\right)$, the Hankel determinant simplifies respectively to

$$
\begin{gathered}
H_{2}(1)=\left|\begin{array}{ll}
a_{1} & a_{2} \\
a_{2} & a_{3}
\end{array}\right|=a_{3}-a_{2}^{2}, \\
\text { and } H_{2}(2)=\left|\begin{array}{ll}
a_{2} & a_{3} \\
a_{3} & a_{4}
\end{array}\right|=a_{2} a_{4}-a_{3}^{2} .
\end{gathered}
$$

We refer to $H_{2}(2)$ as the second Hankel determinant. It is well known that for the univalent functions of the form (1.1), the sharp inequality $H_{2}(1)=\left|a_{3}-a_{2}^{2}\right| \leq 1$ holds true [4]. For a family $\mathcal{T}$ of functions in $S$, the more general problem of finding sharp estimates for the functional $\left|a_{3}-\mu a_{2}^{2}\right|(\mu \in \mathbb{R}$ or $\mu \in \mathbb{C})$, popularly known as the Fekete-Szegö problem for $\mathcal{T}$. Ali [3] found sharp bounds for the first four coefficients and sharp estimate for the Fekete-Szegö functional $\left|\gamma_{3}-t \gamma_{2}^{2}\right|$, where $t$ is real for the inverse function of $f$ (given in (1.1)), defined as $f^{-1}(w)=w+$ $\sum_{n=2}^{\infty} \gamma_{n} w^{n}$ when $f^{-1} \in \widetilde{S T}(\alpha)(0<\alpha \leq 1)$, the class of strongly starlike functions of order $\alpha$. Recently, R. M. Ali, S. K. Lee, V. Ravichandran and S. Supramaniam [2] have obtained sharp bounds for the Fekete-Szegö functional denoted by $\mid b_{2 k+1}-$ $\mu b_{k+1}^{2} \mid$ associated with the $k^{t h}$ root transform $\left[f\left(z^{k}\right)\right]^{\frac{1}{k}}$ of the function given in (1.1) when it belongs to certain subclasses of $S$. The $k^{t h}$ root transform for the function $f$ given in (1.1) is defined as

$$
F(z):=\left[f\left(z^{k}\right)\right]^{\frac{1}{k}}=z+\sum_{n=1}^{\infty} b_{k n+1} z^{k n+1} .
$$

Motivated by the results obtained by R. M. Ali, S. K. Lee, V. Ravichandran and S. Supramaniam [2], in the present paper, we obtain sharp upper bound to the second Hankel determinant denoted by $\left|b_{k+1} b_{3 k+1}-b_{2 k+1}^{2}\right|$ for the $k^{t h}$ root transform for the function $f$ when it belongs to the subclasses namely starlike and convex functions with respect to symmetric points respectively denoted by $S T_{s}$ and $C V_{s}$ of $S$, defined as follows. 
Definition 1.1. A function $f(z) \in A$ is said to be in $S T_{s}$, if it satisfies the condition

$$
\operatorname{Re}\left\{\frac{2 z f^{\prime}(z)}{f(z)-f(-z)}\right\}>0, \quad \forall z \in E .
$$

The class $S T_{s}$ was introduced and studied by Sakaguchi [17]. The concept of starlike functions with respect to symmetric points have been extended to starlike functions with respect to $N$-symmetric points by Ratanchand [16] and Prithvipal Singh [14].

Definition 1.2. A function $f(z) \in A$ is said to be in $C V_{s}$, if it satisfies the condition

$$
\operatorname{Re}\left\{\frac{2\left\{z f^{\prime}(z)\right\}^{\prime}}{z f^{\prime}(z)+z f^{\prime}(-z)}\right\}>0, \quad \forall z \in E .
$$

Some preliminary Lemmas required for proving our results are as follows:

\section{Preliminary Results}

Let $\mathscr{P}$ denote the class of functions consisting of $p$, such that

$$
p(z)=1+c_{1} z+c_{2} z^{2}+c_{3} z^{3}+\ldots=1+\sum_{n=1}^{\infty} c_{n} z^{n},
$$

which are regular in the open unit disc $E$ and satisfy $\operatorname{Rep}(z)>0$, for any $z \in E$. Here $p(z)$ is called the Carathéodory function [4].

Lemma 2.1.([12],[18]) If $p \in \mathscr{P}$, then $\left|c_{k}\right| \leq 2$, for each $k \geq 1$ and the inequality is sharp for the function $\frac{1+z}{1-z}$.

Lemma 2.2.([6]) The power series for $p$ given in (2.1) converges in the open unit disc $E$ to a function in $\mathscr{P}$ if and only if the Toeplitz determinants

$$
D_{n}=\left|\begin{array}{ccccc}
2 & c_{1} & c_{2} & \cdots & c_{n} \\
c_{-1} & 2 & c_{1} & \cdots & c_{n-1} \\
\vdots & \vdots & \vdots & \vdots & \vdots \\
c_{-n} & c_{-n+1} & c_{-n+2} & \cdots & 2
\end{array}\right|, n=1,2,3 \ldots
$$

and $c_{-k}=\bar{c}_{k}$, are all non-negative. They are strictly positive except for $p(z)=$ $\sum_{k=1}^{m} \rho_{k} p_{0}\left(\exp \left(i t_{k}\right) z\right), \rho_{k}>0, t_{k}$ real and $t_{k} \neq t_{j}$, for $k \neq j$, where $p_{0}(z)=\frac{1+z}{1-z}$; in this case $D_{n}>0$ for $n<(m-1)$ and $D_{n} \doteq 0$ for $n \geq m$.

This necessary and sufficient condition found in [6] is due to Carathéodory and Toeplitz. We may assume without restriction that $c_{1}>0$. On using Lemma 2.2, for $n=2$ and $n=3$ respectively, we have

$$
D_{2}=\left|\begin{array}{ccc}
2 & c_{1} & c_{2} \\
\bar{c}_{1} & 2 & c_{1} \\
\bar{c}_{2} & \bar{c}_{1} & 2
\end{array}\right|
$$


On expanding the determinant, we get

$$
D_{2}=\left[8+2 \operatorname{Re}\left\{c_{1}^{2} c_{2}\right\}-2\left|c_{2}\right|^{2}-4\left|c_{1}\right|^{2}\right] \geq 0,
$$

Applying the fundamental principles of complexnumbers, the above expression is equivalent to

$$
2 c_{2}=c_{1}^{2}+x\left(4-c_{1}^{2}\right) \text {, for some } x \text { with }|x| \leq 1 .
$$

$$
\text { and } D_{3}=\left|\begin{array}{cccc}
2 & c_{1} & c_{2} & c_{3} \\
\bar{c}_{1} & 2 & c_{1} & c_{2} \\
\bar{c}_{2} & \bar{c}_{1} & 2 & c_{1} \\
\bar{c}_{3} & \bar{c}_{2} & \bar{c}_{1} & 2
\end{array}\right| \text {. }
$$

Then $D_{3} \geq 0$ is equivalent to

$$
\left|\left(4 c_{3}-4 c_{1} c_{2}+c_{1}^{3}\right)\left(4-c_{1}^{2}\right)+c_{1}\left(2 c_{2}-c_{1}^{2}\right)^{2}\right| \leq 2\left(4-c_{1}^{2}\right)^{2}-2\left|\left(2 c_{2}-c_{1}^{2}\right)\right|^{2} .
$$

Simplifying the relations (2.2) and (2.3), we obtain

$$
\begin{array}{r}
4 c_{3}=c_{1}^{3}+2 c_{1}\left(4-c_{1}^{2}\right) x-c_{1}\left(4-c_{1}^{2}\right) x^{2}+2\left(4-c_{1}^{2}\right)\left(1-|x|^{2}\right) z \\
\text { for some } z, \text { with }|z| \leq 1 .
\end{array}
$$

In obtaining our results, we refer to the classical method devised by Libera and Zlotkiewicz [8].

\section{Main Results}

Theorem 3.1. If $f$ given by (1.1) belongs to $S T_{s}$ and $F$ is the $k^{\text {th }}$ root transformation of $f$ given by (1.2) then

$$
\left|b_{k+1} b_{3 k+1}-b_{2 k+1}^{2}\right| \leq \frac{1}{k^{2}}
$$

and the inequality is sharp.

Proof. Let $f \in S T_{s}$, it follows from the Definition 1.1 there exists an analytic function $p \in \mathscr{P}$ in the open unit disc $E$ with $p(0)=1$ and $\operatorname{Rep}(z)>0$ such that

$$
\frac{2 z f^{\prime}(z)}{f(z)-f(-z)}=p(z) \Leftrightarrow 2 z f^{\prime}(z)=\{f(z)-f(-z)\} p(z) .
$$

Replacing $f(z), f^{\prime}(z), f(-z)$ and $p(z)$ with their equivalent series expressions in (3.1), we have

$$
\begin{aligned}
2 z\left\{1+\sum_{n=2}^{\infty} n a_{n} z^{n-1}\right\} & \\
= & {\left[\left\{z+\sum_{n=2}^{\infty} a_{n} z^{n}\right\}-\left\{-z+\sum_{n=2}^{\infty} a_{n}(-z)^{n}\right\}\right]\left\{1+\sum_{n=1}^{\infty} c_{n} z^{n}\right\} . }
\end{aligned}
$$


Upon simplification, we obtain

$$
2 a_{2} z+3 a_{3} z^{2}+4 a_{4} z^{3}+\ldots=c_{1} z+\left(c_{2}+a_{3}\right) z^{2}+\left(c_{3}+c_{1} a_{3}\right) z^{3}+\ldots
$$

Equating the coefficients of like powers of $z, z^{2}$ and $z^{3}$ respectively on both sides of the relation (3.2), after simplifying, we get

$$
a_{2}=\frac{c_{1}}{2} ; a_{3}=\frac{c_{2}}{2} ; a_{4}=\frac{1}{8}\left(2 c_{3}+c_{1} c_{2}\right)
$$

For the function $f$ given in (1.1), a computation shows that

$$
\begin{aligned}
{\left[f\left(z^{k}\right)\right]^{\frac{1}{k}}=} & {\left[z^{k}+\sum_{n=2}^{\infty} a_{n} z^{n k}\right]^{\frac{1}{k}} } \\
= & {\left[z+\frac{1}{k} a_{2} z^{k+1}+\left\{\frac{1}{k} a_{3}+\frac{1-k}{2 k^{2}} a_{2}^{2}\right\} z^{2 k+1}\right.} \\
& \left.+\left\{\frac{1}{k} a_{4}+\frac{1-k}{k^{2}} a_{2} a_{3}+\frac{(1-k)(1-2 k)}{6 k^{3}} a_{2}^{3}\right\} z^{3 k+1}+\cdots\right] .
\end{aligned}
$$

The equations (1.3) and (3.4) yield;

$$
\begin{aligned}
b_{k+1}=\frac{1}{k} a_{2} ; \quad b_{2 k+1} & =\frac{1}{k} a_{3}+\frac{1-k}{2 k^{2}} a_{2}^{2} ; \\
b_{3 k+1} & =\frac{1}{k} a_{4}+\frac{1-k}{k^{2}} a_{2} a_{3}+\frac{(1-k)(1-2 k)}{6 k^{3}} a_{2}^{3} .
\end{aligned}
$$

Simplifying the expressions (3.3) and (3.5), we get

$$
\begin{aligned}
b_{k+1}=\frac{c_{1}}{2 k} ; \quad b_{2 k+1} & =\frac{c_{2}}{3 k}-\frac{k-1}{8 k^{2}} c_{1}^{2} ; \\
b_{3 k+1} & =\frac{c_{3}}{4 k}-\frac{k-1}{6 k^{2}} c_{1} c_{2}+\frac{(k-1)(2 k-1)}{48 k^{3}} c_{1}^{3} .
\end{aligned}
$$

Substituting the values of $b_{k+1}, b_{2 k+1}$ and $b_{3 k+1}$ from (3.6) in the second Hankel determinant for the $k^{t h}$ - root transformation for the function $f \in S T_{s}$, which simplifies to

$$
\left|b_{k+1} b_{3 k+1}-b_{2 k+1}^{2}\right|=\frac{1}{192 k^{4}}\left|24 k^{2} c_{1} c_{3}+12 k^{2} c_{1}^{2} c_{2}-48 k^{2} c_{2}^{2}-(1-k)(1+4 k) c_{1}^{4}\right| .
$$

From Lemma 2.2, substituting the values of $c_{2}$ and $c_{3}$ from (2.2) and (2.4) respectively, on the right-hand side of the expression (3.7), we have

$$
\begin{aligned}
\left|b_{k+1} b_{3 k+1}-b_{2 k+1}^{2}\right|=\mid 24 k^{2} c_{1} \times \frac{1}{4}\left\{c_{1}^{3}+2 c_{1}\left(4-c_{1}^{2}\right) x-c_{1}\left(4-c_{1}^{2}\right) x^{2}\right. & \\
\left.+2\left(4-c_{1}^{2}\right)\left(1-|x|^{2}\right) z\right\} & +12 k^{2} c_{1}^{2} \times \frac{1}{2}\left\{c_{1}^{2}+x\left(4-c_{1}^{2}\right)\right\} \\
- & 48 k^{2} \times \frac{1}{4}\left\{c_{1}^{2}+x\left(4-c_{1}^{2}\right)\right\}^{2}-(1-k)(1+4 k) c_{1}^{4} \mid .
\end{aligned}
$$


Using the facts $|z|<1$ and $|p a+q b| \leq|p||a|+|q||b|$ where $p, q, a$ and $b$ are real numbers, after simplifying, we get

$$
\begin{aligned}
\left|b_{k+1} b_{3 k+1}-b_{2 k+1}^{2}\right| & \leq \mid-(1-k)(1+4 k) c_{1}^{4}+12 k^{2} c_{1}\left(4-c_{1}^{2}\right) \\
& -6 k^{2} c_{1}^{2}\left(4-c_{1}^{2}\right)|x|-6 k^{2}\left(-c_{1}+4\right)\left(c_{1}+2\right)\left(4-c_{1}^{2}\right)|x|^{2} \mid .
\end{aligned}
$$

Since $c_{1}=c \in[0,2]$, noting that $\left(-c_{1}+a\right)\left(c_{1}+b\right) \geq\left(-c_{1}-a\right)\left(c_{1}-b\right)$, where $a, b \geq 0$, provided $a \geq b$, applying triangle inequality and replacing $|x|$ by $\mu$ on the right-hand side of (3.8), we obtain

$$
\begin{array}{r}
\left|b_{k+1} b_{3 k+1}-b_{2 k+1}^{2}\right| \leq\left[(k-1)(1+4 k) c^{4}+12 k^{2} c\left(4-c^{2}\right)+6 k^{2} c^{2}\left(4-c^{2}\right) \mu\right. \\
\left.+6 k^{2}(-c-4)(c-2)\left(4-c^{2}\right) \mu^{2}\right]=F(c, \mu), \text { for } 0 \leq \mu=|x| \leq 1
\end{array}
$$

$$
\begin{aligned}
& \text { where } F(c, \mu)=(k-1)(1+4 k) c^{4}+12 k^{2} c\left(4-c^{2}\right) \\
& +6 k^{2} c^{2}\left(4-c^{2}\right) \mu+6 k^{2}(-c-4)(c-2)\left(4-c^{2}\right) \mu^{2} .
\end{aligned}
$$

Next, we maximize the $F(c, \mu)$ on the closed region $[0,2] \times[0,1]$. Differentiating $F(c, \mu)$ in (3.10) partially with respect to $\mu$, we get

$$
\frac{\partial F}{\partial \mu}=6 k^{2}\left\{c^{2}+2(-c-4)(c-2) \mu\right\}\left(4-c^{2}\right) .
$$

For $0<\mu<1$, for fixed $c$ with $0<c<2$ and for every $k$, from (3.11), we observe that $\frac{\partial F}{\partial \mu}>0$. Therefore, $F(c, \mu)$ becomes an increasing function of $\mu$ and hence it cannot have a maximum value at any point in the interior of the closed region $[0,2] \times[0,1]$. Further, for fixed $c \in[0,2]$, we have

$$
\max _{0 \leq \mu \leq 1} F(c, \mu)=F(c, 1)=G(c) .
$$

Simplifying the relations (3.10) and (3.12), we obtain

$$
\begin{gathered}
G(c)=(k-1)(1+4 k) c^{4}-48 k^{2} c^{2}+192 k^{2}, \\
G^{\prime}(c)=4(k-1)(1+4 k) c^{3}-96 k^{2} c .
\end{gathered}
$$

From the expression (3.14), we observe that $G^{\prime}(c) \leq 0$ for all values of $c$ in the interval $[0,2]$ and for every $k$. Therefore, $G(c)$ is a monotonically decreasing function of $c$ in the interval $[0,2]$ and hence its maximum value occurs at $c=0$ only, from (3.13), it is given by

$$
\max _{0 \leq c \leq 2} G(0)=192 k^{2} .
$$

From the expressions (3.9) and (3.15), we get

$$
\left|b_{k+1} b_{3 k+1}-b_{2 k+1}^{2}\right| \leq 192 k^{2} .
$$


Simplifying the relations (3.7) and (3.16), we obtain

$$
\left|b_{k+1} b_{3 k+1}-b_{2 k+1}^{2}\right| \leq \frac{1}{k^{2}} .
$$

By setting $c_{1}=c=0$ and selecting $x=1$ in (2.2) and (2.4), we find $c_{2}=2$ and $c_{3}=0$. Using these values in (3.7), we observe that equality is attained, which shows that our result is sharp. For these values, we derive the extremal function, in this case given by

$$
\frac{2 z f^{\prime}(z)}{f(z)-f(-z)}=1+2 z^{2}+2 z^{4}+\ldots=\frac{1+z^{2}}{1-z^{2}} .
$$

This completes the proof of our Theorem 3.1.

Remark. Choosing $k=1$, in (3.17) the result coincides with that of RamReddy and Vamshee Krishna [15].

Theorem 3.2. If $f$ given by (1.1) belongs to $C V_{s}$ and $F$ is the $k^{\text {th }}$ root transformation of $f$ given by (1.2) then

$$
\left|b_{k+1} b_{3 k+1}-b_{2 k+1}^{2}\right| \leq \frac{1}{9 k^{2}}
$$

and the inequality is sharp.

Proof. Let $f(z)=z+\sum_{n=2}^{\infty} a_{n} z^{n} \in C V_{s}$, by virtue of Definition 1.2, we have

$$
\frac{2\left\{z f^{\prime}(z)\right\}^{\prime}}{f^{\prime}(z)+f^{\prime}(-z)}=p(z) \Leftrightarrow 2\left\{z f^{\prime}(z)\right\}^{\prime}=\left\{f^{\prime}(z)+f^{\prime}(-z)\right\} p(z) .
$$

Replacing $f^{\prime}(z), f^{\prime}(-z), f^{\prime \prime}(z)$ and $p(z)$ with their series equivalent expressions in (3.18) and applying the same procedure described in Theorem 3.1, we have

$$
a_{2}=\frac{c_{1}}{4} ; \quad a_{3}=\frac{c_{2}}{6} ; \quad a_{4}=\frac{1}{32}\left(2 c_{3}+c_{1} c_{2}\right) .
$$

From the relations (3.5) and (3.19), a computation shows that

$$
\begin{aligned}
b_{k+1} & =\frac{c_{1}}{4 k} ; b_{2 k+1}=\frac{1}{96 k^{2}}\left\{16 k c_{2}+3(1-k) c_{1}^{2}\right\} \\
b_{3 k+1} & =\frac{1}{384 k^{3}}\left\{12 k^{2}\left(2 c_{3}+c_{1} c_{2}\right)-16 k(k-1) c_{1} c_{2}+(k-1)(2 k-1) c_{1}^{3}\right\} .
\end{aligned}
$$

Substituting the values of $b_{k+1}, b_{2 k+1}$ and $b_{3 k+1}$ from (3.20) in the second Hankel determinant to the $k^{t h}$ transformation for the function $f \in C V_{s}$, upon simplification, we obtain

$$
\begin{aligned}
& \left|b_{k+1} b_{3 k+1}-b_{2 k+1}^{2}\right|= \\
& \frac{1}{9216 k^{4}}\left|144 k^{2} c_{1} c_{3}+72 k^{2} c_{1}^{2} c_{2}-256 k^{2} c_{2}^{2}+3\left(k^{2}-1\right) c_{1}^{4}\right| .
\end{aligned}
$$


The above expression is equivalent to

$$
\begin{aligned}
& \left|b_{k+1} b_{3 k+1}-b_{2 k+1}^{2}\right|=\frac{1}{9216 k^{4}}\left|d_{1} c_{1} c_{3}+d_{2} c_{1}^{2} c_{2}+d_{3} c_{2}^{2}+d_{4} c_{1}^{4}\right|, \\
& \text { where } d_{1}=144 k^{2} ; d_{2}=72 k^{2} ; d_{3}=-256 k^{2} ; d_{4}=3\left(k^{2}-1\right) .
\end{aligned}
$$

From Lemma 2.2, substituting the values of $c_{2}$ and $c_{3}$ from (2.2) and (2.4) respectively, on the right-hand side of the expression (3.22), applying the same procedure described in Theorem 3.1, upon simplification, we obtain

$$
\begin{aligned}
4 \mid d_{1} c_{1} c_{3}+d_{2} c_{1}^{2} c_{2} & +d_{3} c_{2}^{2}+d_{4} c_{1}^{4}|\leq|\left(d_{1}+2 d_{2}+d_{3}+4 d_{4}\right) c_{1}^{4} \\
& +2 d_{1} c_{1}\left(4-c_{1}^{2}\right)+2\left(d_{1}+d_{2}+d_{3}\right) c_{1}^{2}\left(4-c_{1}^{2}\right)|x| \\
& -\left\{\left(d_{1}+d_{3}\right) c_{1}^{2}+2 d_{1} c_{1}-4 d_{3}\right\}\left(4-c_{1}^{2}\right)|x|^{2} \mid .
\end{aligned}
$$

Using the values of $d_{1}, d_{2}, d_{3}$ and $d_{4}$ from (3.23), after simplifying and substituting the calculated values on the right-hand side of (3.24) and applying the same procedure described in Theorem 3.1, we get

$$
\begin{aligned}
\left|d_{1} c_{1} c_{3}+d_{2} c_{1}^{2} c_{2}+d_{3} c_{2}^{2}+d_{4} c_{1}^{4}\right| \leq\left[\left(11 k^{2}-3\right) c^{4}+\left\{72 k^{2} c+20 k^{2} c^{2} \mu+\right.\right. & \\
\left.4 k^{2}(-7 c-32)(c-2) \mu^{2}\right\} & \left.\left(4-c^{2}\right)\right] \\
& =F(c, \mu), \text { for } 0 \leq \mu=|x| \leq 1
\end{aligned}
$$

$$
\begin{aligned}
\text { where } F(c, \mu)=\left(11 k^{2}-3\right) c^{4}+\left\{72 k^{2} c\right. & +20 k^{2} c^{2} \mu \\
& \left.+4 k^{2}(-7 c-32)(c-2) \mu^{2}\right\}\left(4-c^{2}\right) .
\end{aligned}
$$

Applying the same procedure described in Theorem 3.1, we observe that $\frac{\partial F}{\partial \mu}>0$. Therefore, $F(c, \mu)$ becomes an increasing function of $\mu$. Moreover, for fixed $c \in[0,2]$, we have

$$
\max _{0 \leq \mu \leq 1} F(c, \mu)=F(c, 1)=G(c) .
$$

In view of (3.27), replacing $\mu$ by 1 in (3.26), which simplifies to

$$
\begin{gathered}
G(c)=\left(19 k^{2}-3\right) c^{4}-288 k^{2} c^{2}+1024 k^{2}, \\
G^{\prime}(c)=4\left(19 k^{2}-3\right) c^{3}-576 k^{2} c .
\end{gathered}
$$

From the expression (3.29), we observe that $G^{\prime}(c) \leq 0$ for every $c \in[0,2]$ and for all values of $k$ so that the maximum value of $G(c)$ occurs at $c=0$ only, from (3.28), it is given by

$$
\max _{0 \leq c \leq 2} G(0)=1024 k^{2}
$$


Simplifying the relations (3.22) and (3.25) along with (3.30), we get

$$
\left|b_{k+1} b_{3 k+1}-b_{2 k+1}^{2}\right| \leq \frac{1}{9 k^{2}}
$$

If we set $c_{1}=c=0$ and choosing $x=1$ in (2.2) and (2.4), we find that $c_{2}=2$ and $c_{3}=0$ respectively. Using these values in (3.21), we observe that equality is attained, which shows that our result is sharp. For these values, we derive the extremal function as

$$
\frac{2\left\{z f^{\prime}(z)\right\}^{\prime}}{f^{\prime}(z)+f^{\prime}(-z)}=1+2 z^{2}+2 z^{4}+\ldots=\frac{1+z^{2}}{1-z^{2}} .
$$

This completes the proof of our second Theorem 3.2.

Remark. Choosing $k=1$ in (3.31), it coincides with that of RamReddy and Vamshee Krishna [15].

Acknowledgements. The authors would like to express sincere thanks to the esteemed Referee(s) for their careful readings, valuable suggestions and comments, which helped them to improve the presentation of this paper.

\section{References}

[1] A. Abubaker and M. Darus, Hankel Determinant for a class of analytic functions involving a generalized linear differential operator, Int. J. Pure Appl. Math., 69(4)(2011), 429 - 435.

[2] R. M. Ali, S. K. Lee, V. Ravichandran and S. Supramaniam, The Fekete-Szegö coefficient functional for transforms of analytic functions, Bull. Iran. Math. Soc., 35(2)(2009), 119-142.

[3] R. M. Ali, Coefficients of the inverse of strongly starlike functions, Bull. Malays. Math. Sci. Soc., (second series), 26(1)(2003), 63 - 71.

[4] P. L. Duren, Univalent functions, 259 Grundlehren der Mathematischen Wissenschaften, New York, Springer-verlag XIV, 328, 1983.

[5] R. Ehrenborg, The Hankel determinant of exponential polynomials, Amer. Math. Monthly, 107(6)(2000), 557 - 560.

[6] U. Grenander and G. Szego, Toeplitz forms and their applications. 2nd ed. New York (NY): Chelsea Publishing Co., 1984.

[7] A. Janteng, S. A. Halim and M. Darus, Hankel determinant for starlike and convex functions, Int. J. Math. Anal., (Ruse) 4(13-16)(2007), 619 - 625.

[8] R. J. Libera and E. J. Zlotkiewicz, Coefficient bounds for the inverse of a function with derivative in $\mathscr{P}$, Proc. Amer. Math. Soc., 87(1983), 251-257. 
[9] A. K. Mishara and S. N. Kund, The second Hankel determinant for a class of analytic functions associated with the carlson-shaffer operator, Tamkang J. Math., 44(1)(2013), 73 - 82.

[10] N. Mohamed, D. Mohamad and S. Cik Soh, Second Hankel determinant for certain generalized classes of analytic functions, Int. J. Math. Anal., (Ruse) 6(17-20)(2012), $807-812$.

[11] K. I. Noor, Hankel determinant problem for the class of functions with bounded boundary rotation, Rev. Roum. Math. Pures Et Appl., 28(8)(1983), 731 - 739.

[12] Ch. Pommerenke, Univalent functions, Gottingen: Vandenhoeck and Ruprecht, 1975.

[13] Ch. Pommerenke, On the coefficients and Hankel determinants of univalent functions, J. Lond. Math. Soc., 41(1966), 111 - 122.

[14] Prithvipal Singh, A study of some subclasses of analytic functions in the unit disc, Ph. D Thesis, 1979, IIT Kanpur.

[15] T. RamReddy and D. Vamshee Krishna, Hankel Determinant for starlike and convex functions with respect to symmetric points, J. Ind. Math. Soc., 79(1-4)(2012), 161 171.

[16] Ratanchand, Some aspects of functions analytic in the unit disc, $\mathrm{Ph} . \mathrm{D}$ Thesis 1978, IIT Kanpur.

[17] K. Sakaguchi, On a certain univalent mapping, J. Math. Soc. Japan, 11(1959), 72 75 .

[18] B. Simon, Orthogonal polynomials on the unit circle, part 1. Classical theory. Vol.54, American mathematical society colloquium publications. Providence (RI): American Mathematical Society, 2005. 